\title{
On Adaptive Output Feedback Controf Robotic Manipulators with Online Disturbance Estimation
}

\author{
Orhan Aksoy • Erkan Zergeroglu • \\ Enver Tatlicioglu
}

Received: 1 September 2015 / Accepted: 11 July 2016 / Published online: 21 July 2016

(C) Springer Science+Business Media Dordrecht 2016

\begin{abstract}
The problem of disturbance estimation and compensation for adaptive output feedback type controllers are investigated. Specifically two adaptive output feedback controllers designed for robotic manipulators are extended to compensate external disturbances which are common in robotic applications with repetitive task. The uncertain disturbance term in the robot dynamics is modeled as a fixed term plus a combination of sinusoidal signals. The overall stability and convergence of the tracking error for both controllers is ensured via Lyapunov based analysis. Extensive simulation studies are presented to illustrate the feasibility of the proposed method.
\end{abstract}

Keywords Adaptive control · Output feedback control $\cdot$ Robotic manipulators

\section{O. Aksoy}

HAVELSAN A.S., DSYSTM, Pendik, Istanbul 34890 ,

Turkey

e-mail: aksoy@ havelsan.com.tr

\section{E. Zergeroglu $(\bowtie)$}

Department of Computer Engineering, Gebze Technical University, 41400 Gebze, Kocaeli, Turkey

e-mail: ezerger@bilmuh.gyte.edu.tr

\section{E. Tatlicioglu}

Department of Electrical \& Electronics Engineering, Izmir Institute of Technology, Gulbahce Koyu, Urla, Izmir, 35430 Turkey

e-mail: envertatlicioglu@iyte.edu.tr

\section{Introduction}

Output feedback control ${ }^{1}$ of nonlinear systems modeled by Euler-Lagrange formulation have attracted considerable attention from researchers, especially when the system under consideration exhibits uncertainties in their dynamics. For robotic manipulators, this means the control formulation is required to use only the link position measurements. Considering that most commercial robotic manipulators do not have velocity sensors and external sensors used for velocity measurements are mostly contaminated with noise, the output feedback control problem of robotic manipulators is well motivated. One standard method to eliminate the need of velocity measurements is to apply numerical differentiation plus low pass filtering on position measurements [1]. This method also known as "backwards difference" may, on some occasions, provide reasonable performance, however from a theoretical point of view inserts an extra filter dynamics in to the closed loop system.

To our best knowledge the output feedback controller formulations presented in the literature can be categorized as observer based [2-4], and filter based [5-7] formulations. In most observer based methods, a model based observer is used to estimate the velocity signal, where in filter based approaches surrogate

\footnotetext{
${ }^{1}$ Among the system states only the output, i.e., the position measurements are available for the controller formulation.
} 
filters are used to overcome velocity measurement requirements [4].

On the other hand, when the dynamical parameters of the robotic system are not precisely known, one way to compensate for the uncertainties is to use adaptive type controllers. The main drawback of adaptive type controllers is the requirement of so called "structured uncertainties". That is; the stability proof of adaptive type controllers requires the unknown system parameters to appear linearly in the dynamical model. However, this linear parameterization requirement is not applicable to most Euler-Lagrange systems, as robotic manipulators, due to the frictional effects, unmodeled dynamical terms and external disturbances [8]. To remove this weakness, robustifying or artificial intelligence based terms have been used in conjunction with adaptive controllers [8]. In [9-11], adaptive fuzzy sliding mode controllers have been proposed to control electrical servo systems where the fuzzy system was used to approximate the unknown nonlinear parts of the model of the controlled process like a feedback linearizing term, and the sliding mode controller was as a feed-forward controller. In [8], a desired compensation adaptive law including a periodic disturbance estimation mechanism has been applied to overcome this problem. But the proposed solutions utilized full system state information in the controller designs. A model based observer-controller structure that achieved semi-global exponential position tracking result was presented in [12]. Similarly robust filtered-based controllers that produced semiglobal uniformly ultimately bounded position tracking were introduced in [13] and [14].

In an attempt to address the output feedback control of Euler-Lagrange systems subject to both structured (i.e., linearly parameterized) and unstructured (i.e., unmodeled dynamics, disturbances and nonlinearly parameterized) uncertainties, in this paper, we present two adaptive output feedback controllers for robotic manipulators with a novel disturbance estimation technique. For both controllers the structured uncertainties, similar to standard adaptive controller approaches, are compensated via a dynamic parameter update term, and the unstructured uncertainties of the dynamics are compensated via a modified version of the periodic disturbance estimator of [8]. In order to illustrate the flexibility of the proposed methodology, two different types of adaptive output feedback controllers; first a filter based, then an observer based controller formulations are presented. The filter based controller formulation is an extension of the global adaptive output feedback controller presented in [5] and the observer based formulation is an extension of the adaptive controller of [4]. Specifically, we have extended the results given in [5] and [4] so that both controller formulations can also compensate unstructured uncertainties in the robot dynamics. For both formulations stability of the closed loop systems are ensured via Lyapunov based analysis and extensive numerical simulations are performed on the model of a two link robot manipulator to illustrate the effectiveness of the proposed methods.

The rest of the paper is organized as follows. In Section 2, robot dynamics and model properties that are used in the analysis and design of the proposed controllers are presented. Section 3 contains the problem formulation. In Section 4, error system developments, controller designs and stability analysis of both approaches are presented. Section 5 contains numerical simulation studies for both controllers applied to a two link direct drive planar robot manipulator with additive external disturbances. Finally Section 6 contains some concluding remarks.

\section{System Dynamics and Properties}

The dynamical model of an $n$ - degree of freedom (DOF) robotic manipulator modeled by EulerLagrange formulation is assumed to have the following form

$$
M(q) \ddot{q}+V_{m}(q, \dot{q}) \dot{q}+G(q)+F_{d} \dot{q}+\tau_{d}=\tau
$$

where $q(t), \dot{q}(t), \ddot{q}(t) \in \mathbb{R}^{n}$ represent position, velocity and acceleration, respectively, $M(q) \in \mathbb{R}^{n \times n}$ represents the inertia matrix, $V_{m}(q, \dot{q}) \in \mathbb{R}^{n \times n}$ represents the centripetal-Coriolis matrix, $G(\dot{q}) \in \mathbb{R}^{n}$ represents the gravitational effects, the constant diagonal matrix $F_{d} \in \mathbb{R}^{n \times n}$ represents the viscous frictional effects, $\tau_{d}(t) \in \mathbb{R}^{n}$ represents additive disturbance effects and unmodeled terms, and $\tau(t) \in \mathbb{R}^{n}$ represents the control input. Euler-Lagrange systems exhibit the following properties that will be used in the subsequent control development and analysis sections.

Property 1 The inertia matrix can be bounded from above and below by the following inequalities $[15,16]$

$m_{1} I_{n} \leq M(q) \leq m_{2} I_{n}$ 
where $m_{1}$ and $m_{2}$ are positive constants and $I_{n}$ is the standard $n \times n$ identity matrix. Likewise, the inverse of the inertia matrix can be bounded from above and below as follows

$\frac{1}{m_{2}} I_{n} \leq M^{-1}(q) \leq \frac{1}{m_{1}} I_{n}$.

Property 2 The inertia and the centripetal-Coriolis matrices satisfy the following skew-symmetry relationship [17]

$\xi^{T}\left(\frac{1}{2} \dot{M}(q)-V_{m}(q, \dot{q})\right) \xi=0 \quad \forall \xi \in \mathbb{R}^{n}$

where $\dot{M}(q)$ represents the time derivative of the inertia matrix.

Property 3 The centripetal-Coriolis matrix satisfies the following relationship [18]

$V_{m}(q, v) \xi=V_{m}(q, \xi) v \quad \forall \xi, v \in \mathbb{R}^{n}$

Property 4 The norms of the centripetal-Coriolis and friction matrices can be upper bounded as follows [15]

$$
\left\|V_{m}(q, \xi)\right\|_{i \infty} \leq \zeta_{c 1}\|\xi\|, \quad\left\|F_{d}\right\|_{i \infty} \leq \zeta_{f} \quad \forall \xi \in \mathbb{R}^{n}
$$

where $\zeta_{c 1}$ and $\zeta_{f}$ are positive constants and $\|\cdot\|_{i \infty}$ denotes an induced matrix norm.

Property 5 The left-hand side of the dynamics in Eq. 1 can be segregated as the sum of linearly parameterized terms and unstructured terms as follows [8, 15]

$$
\begin{aligned}
Y(q, \dot{q}, \ddot{q}) \theta+\gamma= & M(q) \ddot{q}+V_{m}(q, \dot{q}) \dot{q}+G(q) \\
& +F_{d} \dot{q}+\tau_{d}
\end{aligned}
$$

where $\theta \in \mathbb{R}^{p}$ contains the constant system parameters, $Y(q, \dot{q}, \ddot{q}) \in \mathbb{R}^{n \times p}$ denotes the regression matrix that is a function of only $q(t), \dot{q}(t)$ and $\ddot{q}(t)$, and $\gamma(t) \in \mathbb{R}^{n}$ represents the unstructured part of the unmodeled dynamics. The formulation of Eq. 7 can also be written in terms of the desired counterparts of the system states in the following manner

$$
\begin{aligned}
Y_{d}\left(q_{d}, \dot{q}_{d}, \ddot{q}_{d}\right) \theta+\gamma= & M\left(q_{d}\right) \ddot{q}_{d}+V_{m}\left(q_{d}, \dot{q}_{d}\right) \dot{q}_{d} \\
& +G\left(q_{d}\right)+F_{d} \dot{q}_{d}+\tau_{d}
\end{aligned}
$$

where the desired regression matrix $Y_{d}\left(q_{d}, \dot{q}_{d}, \ddot{q}_{d}\right) \in$ $\mathbb{R}^{n \times p}$ is a function of the desired position, velocity, and acceleration vectors denoted by $q_{d}(t), \dot{q}_{d}(t)$, $\ddot{q}_{d}(t) \in \mathbb{R}^{n}$, respectively.

Property 6 The inertia, centripetal-Coriolis and gravity terms in Eq. 1 can be upper bounded as follows [19]

$$
\begin{aligned}
\|M(\xi)-M(v)\|_{i \infty} & \leq \zeta_{m 1}\|\xi-v\| \\
\left\|M^{-1}(\xi)-M^{-1}(v)\right\|_{i \infty} & \leq \zeta_{m 2}\|\xi-v\| \\
\left\|V_{m}(\xi, \omega)-V_{m}(\nu, \omega)\right\|_{i \infty} & \leq \zeta_{c 2}\|\xi-v\|\|\omega\| \\
\|G(\xi)-G(v)\| & \leq \zeta_{g}\|\xi-v\|
\end{aligned}
$$

$\forall \nu, \xi, \omega \in \mathbb{R}^{n}$ where $\zeta_{m 1}, \zeta_{m 2}, \zeta_{c 2}, \zeta_{g}$ are positive constants.

\section{Problem Formulation}

The control objective is to design an adaptive tracking controller for the system using only position vector $q(t)$ as the available system state, despite the presence of uncertain system parameters and additive periodic disturbance. We will quantify the control objective by defining the position tracking error, denoted by $e(t) \in$ $\mathbb{R}^{n}$, as follows

$e \triangleq q_{d}-q$.

The desired position and its time derivatives are assumed to be bounded functions of time.

In order to compensate for the uncertain system parameter vector $\theta$, a dynamic estimate, denoted by $\hat{\theta}(t) \in \mathbb{R}^{p}$, will be developed in the subsequent controller designs. The difference between actual and estimated parameters, denoted by $\tilde{\theta}(t) \in \mathbb{R}^{p}$, is defined as

$\tilde{\theta} \triangleq \theta-\hat{\theta}$.

The unstructured disturbance term $\gamma$ defined in Eq. 7, similar to [8], is assumed to be in the following form

$\gamma=E \operatorname{Tanh}(e)+\sum_{k=1}^{h} D_{k} \operatorname{Sin}(k e)$

where $E, D_{k} \in \mathbb{R}^{n \times n}$ for $k=1,2, \ldots, h$ are constant unknown diagonal matrices with the diagonal entries of $E$ standing for the mean value of the disturbance terms and the diagonal entries of $D_{k}$ standing for contributions of different error frequencies to the 
disturbance model, and $h \in \mathbb{Z}^{+}$is the harmonic limit for the approximation. The constant matrices $E$ and $D_{k}$ for $k=1,2, \ldots, h$ are of the form

$$
\begin{aligned}
E & =\operatorname{diag}\left\{E_{1}, E_{2}, . ., E_{n}\right\} \\
D_{k} & =\operatorname{diag}\left\{D_{k 1}, D_{k 2}, . ., D_{k n}\right\}
\end{aligned}
$$

and $\operatorname{Tanh}(e), \operatorname{Sin}(k e) \in \mathbb{R}^{n}$ are vector functions of the tracking error and are defined as

$$
\begin{aligned}
& \operatorname{Tanh}(e) \triangleq\left[\begin{array}{lll}
\tanh \left(e_{1}\right) & \tanh \left(e_{2}\right) & \ldots \\
\tanh \left(e_{n}\right)
\end{array}\right]^{T} \\
& \operatorname{Sin}(k e) \triangleq\left[\begin{array}{llll}
\sin \left(k e_{1}\right) & \sin \left(k e_{2}\right) & \ldots & \sin \left(k e_{n}\right)
\end{array}\right]^{T} .
\end{aligned}
$$

Remark 1 The main idea behind the structure of $\gamma$ given above is to model the external disturbances as a fixed term, defined by the $E \operatorname{Tanh}(e)$ term, plus additional periodic terms represented by the $\sum_{k=1}^{h} D_{k} \operatorname{Sin}(k e)$ part. Notice that both terms are equal to zero when the tracking error is driven to zero. Therefore the proposed controllers would not excite the system unnecessarily, when the tracking objective is achieved.

\section{Controller Design}

Since velocity measurements are not available for controller design, two approaches are utilized to generate a placeholder for the lack of this information. The first approach is based on a nonlinear filter [5] and the second approach is based on a nonlinear observer [4]. We would like to point out that both controller implementations make use of the desired version of the regression matrix $Y_{d}\left(q_{d}, \dot{q}_{d}, \ddot{q}_{d}\right)$ and its time derivative. To ensure this the desired position profile $q_{d}(t)$ must be selected to be at least third-order differentiable.

\subsection{Filter Based Approach}

Based on the subsequent error system development and stability analysis, the control input is designed as follows

$\tau=Y_{d} \hat{\theta}-k_{f} T^{-1} y+\operatorname{Tanh}(e)+\hat{\gamma}$

with the disturbance estimation term $\hat{\gamma}$ designed in the following form

$\hat{\gamma}=\hat{E} \operatorname{Tanh}(e)+\sum_{k=1}^{h} \hat{D}_{k} \operatorname{Sin}(k e)$ where $k_{f} \in \mathbb{R}$ is a positive gain, $\hat{E}(t), \hat{D}_{k}(t) \in \mathbb{R}^{n \times n}$ for $k=1,2, \ldots, h$ represent the subsequently designed dynamic estimates of $E, D_{k}$, respectively, $T(y) \in$ $\mathbb{R}^{n \times n}$ is a diagonal matrix defined as

$T=\operatorname{diag}\left\{\left(1-y_{1}^{2}\right)^{2}\left(1-y_{2}^{2}\right)^{2} \ldots\left(1-y_{n}^{2}\right)^{2}\right\}$

with $y(t) \triangleq\left[\begin{array}{llll}y_{1} & y_{2} & \ldots & y_{n}\end{array}\right]^{T} \in \mathbb{R}^{n}$ being an auxiliary filter-like term with its entries obtained from

$y_{i}=p_{i}-k_{f} e_{i}$

where $p(t) \in \mathbb{R}^{n}$ is another auxiliary filter-like term with its entries $p_{i}(t) \in \mathbb{R}$ updated according to

$$
\begin{aligned}
\dot{p}_{i}= & -\left(1-\left(p_{i}-k_{f} e_{i}\right)^{2}\right)^{2}\left(p_{i}-k_{f} e_{i}-\tanh \left(e_{i}\right)\right) \\
& -k_{f}\left(\tanh \left(e_{i}\right)+p_{i}-k_{f} e_{i}\right)
\end{aligned}
$$

with initial conditions satisfying

$-\frac{1}{\sqrt{n}}+k_{f} e_{i}(0)<p_{i}(0)<\frac{1}{\sqrt{n}}+k_{f} e_{i}(0)$.

Remark 2 From the structure of (21) it is evident that for the implementation of the controller the inverse of the matrix $T$ need to be calculated on line. Notice that from the structure of (22) that the entries of the $T$ matrix is always positive and therefore it is always possible to calculate its inverse.

The adaptive update rules for $\hat{\theta}(t), \hat{E}(t), \hat{D}_{k}(t)$ are generated according to

$$
\begin{aligned}
\hat{\theta}(t)=\Gamma \int_{0}^{t}\left[Y_{d}^{T}(\sigma)(\operatorname{Tanh}(e(\sigma))+y(\sigma))\right. & \left.-\frac{d Y_{d}^{T}(\sigma)}{d \sigma} e(\sigma)\right] d \sigma+\Gamma Y_{d}^{T}(t) e(t) \\
& -\Gamma Y_{d}^{T}(0) e(0) \\
\hat{E}(t)= & \Psi \int_{0}^{t} v_{f}(\sigma) d_{\tanh }(\sigma) d \sigma+\Psi d_{\ln }(t)-\Psi d_{\ln }(0)
\end{aligned}
$$

$$
\begin{aligned}
\hat{D}_{k}(t)= & \Psi_{k} \int_{0}^{t} v_{f}(\sigma) d_{\sin }(\sigma) d \sigma-\frac{1}{k} \Psi_{k} d_{\cos }(t) \\
& +\frac{1}{k} \Psi_{k} d_{\cos }(0) \text { for } k=1,2, \ldots, h
\end{aligned}
$$


where $d_{\mathrm{tanh}}(t), d_{\cos }(t), d_{\sin }(t), d_{\ln }(t), v_{f}(t) \in \mathbb{R}^{n \times n}$ are defined as

$$
\begin{aligned}
d_{\tanh }(t) & \triangleq \operatorname{diag}\left\{\tanh \left(e_{1}(t)\right), \tanh \left(e_{2}(t)\right), . ., \tanh \left(e_{n}(t)\right)\right\} \\
d_{\cos }(t) & \triangleq \operatorname{diag}\left\{\cos \left(k e_{1}(t)\right), \cos \left(k e_{2}(t)\right), . ., \cos \left(k e_{n}(t)\right)\right\} \\
d_{\sin }(t) & \triangleq \operatorname{diag}\left\{\sin \left(k e_{1}(t)\right), \sin \left(k e_{2}(t)\right), . ., \sin \left(k e_{n}(t)\right)\right\} \\
d_{\ln }(t) & \triangleq \operatorname{diag}\left\{\ln \left(\cosh \left(e_{1}(t)\right)\right), \ln \left(\cosh \left(e_{2}(t)\right)\right), . ., \ln \left(\cosh \left(e_{n}(t)\right)\right)\right\} \\
v_{f}(t) & \triangleq \operatorname{diag}\left\{\tanh \left(e_{1}(t)\right)+y_{1}(t), \tanh \left(e_{2}(t)\right)+y_{2}(t), . ., \tanh \left(e_{n}(t)\right)+y_{n}(t)\right\}
\end{aligned}
$$

with $\Gamma \in \mathbb{R}^{p \times p}, \Psi \in \mathbb{R}^{n \times n}, \Psi_{k} \in \mathbb{R}^{n \times n} k=$ $1,2, \ldots, h$ being constant, diagonal, positive definite adaptation gain matrices.

To simplify the presentation of the derivation of closed-loop error dynamics, a filtered tracking error, denoted by $\eta(t) \in \mathbb{R}^{n}$, is defined as

$\eta \triangleq \dot{e}+\operatorname{Tanh}(e)+y$.

The dynamics for $y(t)$ is obtained from the time derivative of Eq. 23 as

$\dot{y}_{i}=-\left(1-y_{i}^{2}\right)^{2}\left(y_{i}-\tanh \left(e_{i}\right)\right)-k_{f} \eta_{i}$

where Eqs. 22, 24 and 34 were substituted. The dynamics of the filtered tracking error $\eta(t)$ is then obtained by differentiating (34) and utilizing system dynamics in Eq. 1 and dynamics of $y(t)$ in Eq. 35

$$
\begin{aligned}
M(q) \dot{\eta}= & M(q) \ddot{q}_{d}+V_{m}(q, \dot{q}) \dot{q}+F_{d} \dot{q}+G(q)+\tau_{d}-\tau \\
& +M(q) \operatorname{Cosh}^{-2}(e)(\eta-\operatorname{Tanh}(e)-y) \\
& -M(q) T(y-\operatorname{Tanh}(e))-k_{f} M(q) \eta .
\end{aligned}
$$

Desired form of the dynamics given in Eq. 8 and $V_{m}(q, \dot{q}) \eta$ is added to and subtracted from Eq. 36 to obtain

$$
M(q) \dot{\eta}=-V_{m}(q, \dot{q}) \eta+Y_{d} \theta+\gamma-k_{f} M(q) \eta-\tau+\tilde{X}+\tilde{Y}
$$

where Eqs. 13 and 34 were utilized and $\tilde{X}(t), \tilde{Y}(t) \in$ $\mathbb{R}^{n}$ are defined as

$$
\begin{aligned}
\tilde{X} \triangleq & M(q) \operatorname{Cosh}^{-2}(e)(\eta-\operatorname{Tanh}(e)-y) \\
& -M(q) T(y-\operatorname{Tanh}(e)) \\
& +V_{m}\left(q, \dot{q}_{d}+\operatorname{Tanh}(e)+y\right)(\operatorname{Tanh}(e)+y) \\
& +V_{m}\left(q, \dot{q}_{d}\right)(\operatorname{Tanh}(e)+y) \\
& -V_{m}(q, \eta)\left(\dot{q}_{d}+\operatorname{Tanh}(e)+y\right)
\end{aligned}
$$

$$
\begin{aligned}
\tilde{Y} \triangleq & M(q) \ddot{q}_{d}+V_{m}\left(q, \dot{q}_{d}\right) \dot{q}_{d}+G(q)+F_{d} \dot{q} \\
& -M\left(q_{d}\right) \ddot{q}_{d}-V_{m}\left(q_{d}, \dot{q}_{d}\right) \dot{q}_{d}-G\left(q_{d}\right)-F_{d} \dot{q}_{d}
\end{aligned}
$$

The above auxiliary terms can be bounded as [5]

$$
\begin{aligned}
\|\tilde{X}\| \leq & \xi_{1}\|x\|+\xi_{2}\|y\|^{2}+\xi_{3}\|y\|^{3}+\xi_{4}\|y\|^{4} \\
& +\xi_{5}\|y\|^{5}+\xi_{6}\|\eta\|\|y\| \\
\|\tilde{Y}\| \leq & \xi_{7}\|x\|
\end{aligned}
$$

where $\xi_{i} i=1, \cdots, 7$ are known positive bounding constants and $x(t) \in \mathbb{R}^{3 n}$ is defined as

$x \triangleq\left[\begin{array}{ll}\eta^{T} & y^{T} \operatorname{Tanh}^{T}(e)\end{array}\right]^{T}$.

Finally, substituting the control law in Eq. 20 and the disturbance model in Eq. 15 into the open-loop error system in Eq. 37 gives the following closed-loop error system

$$
\begin{aligned}
M(q) \dot{\eta}= & -V_{m}(q, \dot{q}) \eta-k_{f} M(q) \eta+\tilde{X}+\tilde{Y} \\
& +k_{f} T^{-1} y-\operatorname{Tanh}(e)+Y_{d} \tilde{\theta}+\tilde{E} \operatorname{Tanh}(e) \\
& +\sum_{k=1}^{h} \tilde{D}_{k} \operatorname{Sin}(k e)
\end{aligned}
$$

where $\tilde{E}(t), \tilde{D}_{k}(t) \in \mathbb{R}^{n \times n}$ represent the estimation errors and are defined as

$$
\begin{aligned}
\tilde{E} & \triangleq E-\hat{E} \\
\tilde{D}_{k} & \triangleq D_{k}-\hat{D}_{k} \text { fork }=1,2, \ldots, h .
\end{aligned}
$$

Theorem 1 The controller given in Eq. 20 with adaptive update rules in Eqs. 26-28 ensures global stability of the equilibrium $\left\{\eta=0_{n \times 1}, y=0_{n \times 1}, e=0_{n \times 1}\right.$, $\left.\tilde{\theta}=0_{p \times 1}, v_{\tilde{E}}=0_{n \times 1}, v_{\tilde{D}_{k}}=0_{h n \times 1}\right\}$ where

$$
\begin{aligned}
& v_{\tilde{E}}=\left[\begin{array}{llll}
\tilde{E}_{1} & \tilde{E}_{2} & \ldots & \tilde{E}_{n}
\end{array}\right]^{T} \in \mathbb{R}^{n} \\
& v_{\tilde{D}_{k}}=\left[\begin{array}{llllllllll}
\tilde{D}_{11} & \ldots & \tilde{D}_{1 n} & \tilde{D}_{21} & \ldots & \tilde{D}_{2 n} & \tilde{D}_{h 1} & \ldots & \tilde{D}_{h n}
\end{array}\right]^{T} \in \mathbb{R}^{h n}
\end{aligned}
$$


and convergence to the set $\left\{\eta=0_{n \times 1}, y=0_{n \times 1}\right.$, $\left.e=0_{n \times 1}\right\}$ in the region

$$
\begin{gathered}
S_{1}=\left\{( \eta , y , e , \tilde { \theta } , v _ { \tilde { E } } , v _ { \tilde { D } _ { k } } ) \in \left(\mathbb{R}^{n},\left(-\frac{1}{\sqrt{n}}, \frac{1}{\sqrt{n}}\right)^{n},\right.\right. \\
\left.\left.\mathbb{R}^{n}, \mathbb{R}^{p}, \mathbb{R}^{n}, \mathbb{R}^{h n}\right)\right\}
\end{gathered}
$$

provided the controller gain $k_{f}$ is selected as

$$
\begin{gathered}
k_{f}=\frac{1}{m_{1}}\left(1+k_{n}\left(\xi_{1}+\xi_{7}\right)^{2}+2 n \xi_{2}^{2}+2 n^{2} \xi_{3}^{2}\right. \\
\left.+2 n^{3} \xi_{4}^{2}+2 n^{4} \xi_{5}^{2}+\xi_{6} \sqrt{n}\right)
\end{gathered}
$$

where $m_{1}$ was defined in Eq. 2, $\xi_{i} i=1, \ldots, 7$ were defined in Eq. 40 and $k_{n}$ is a nonlinear damping gain.

Proof To prove the above theorem, a non-negative scalar function, denoted by $V_{f}(t) \in \mathbb{R}$, is defined

$$
\begin{aligned}
V_{f} \triangleq & \frac{1}{2} \eta^{T} M(q) \eta+\frac{1}{2} \sum_{i=1}^{n} \frac{y_{i}^{2}}{1-y_{i}^{2}}+\sum_{i=1}^{n} \ln \left(\cosh \left(e_{i}\right)\right) \\
& +\frac{1}{2} \tilde{\theta}^{T} \Gamma^{-1} \tilde{\theta}+\frac{1}{2} \operatorname{tr}\left\{\tilde{E} \Psi^{-1} \tilde{E}\right\} \\
& +\frac{1}{2} \operatorname{tr}\left\{\sum_{k=1}^{h} \tilde{D}_{k} \Psi_{k}^{-1} \tilde{D}_{k}\right\}
\end{aligned}
$$

where $\operatorname{tr}\{\cdot\}$ represents the trace of a square matrix. In Eq. $50, \ln \left(\cosh \left(e_{i}\right)\right)$ is positive definite and radially unbounded, and $\frac{y_{i}^{2}}{1-y_{i}^{2}}$ is positive definite and radially unbounded in the region $y_{i} \in[-1,1]$, as a result, $V_{f}(t)$ is positive definite and radially unbounded function in the set

$$
\begin{gathered}
S=\left\{( \eta , y , e , \tilde { \theta } , v _ { \tilde { E } } , v _ { \tilde { D } _ { k } } ) \in \left(\mathbb{R}^{n},[-1,1]^{n}, \mathbb{R}^{n}, \mathbb{R}^{p},\right.\right. \\
\left.\left.\mathbb{R}^{n}, \mathbb{R}^{h n}\right)\right\} .
\end{gathered}
$$

Taking the time derivative of Eq. 50 along the error dynamics in Eqs. 34, 35, 43, and then simplifying results in

$$
\begin{aligned}
\dot{V}_{f}= & -k_{f} \eta^{T} M(q) \eta+\eta^{T}(\tilde{X}+\tilde{Y})-\sum_{i=1}^{n} y_{i}^{2} \\
& -\sum_{i=1}^{n} \tanh ^{2}\left(e_{i}\right)
\end{aligned}
$$

where the time derivatives of Eqs. 26-28 were utilized as

$$
\begin{aligned}
\dot{\hat{\theta}} \triangleq & \Gamma Y_{d}^{T} \eta \\
\dot{\hat{E}} \triangleq & \Psi \operatorname{diag}\left\{\eta_{1} \tanh \left(e_{1}\right), \eta_{2} \tanh \left(e_{2}\right), \cdots,\right. \\
& \left.\eta_{n} \tanh \left(e_{n}\right)\right\} \\
\dot{\hat{D}}_{k} \triangleq & \Psi_{k} \operatorname{diag}\left\{\eta_{1} \sin \left(k e_{1}\right), \eta_{2} \sin \left(k e_{2}\right), \cdots,\right. \\
& \left.\eta_{n} \sin \left(k e_{n}\right)\right\} .
\end{aligned}
$$

After utilizing the bounds for $M(q), \tilde{X}(t)$ and $\tilde{Y}(t)$ given in Eqs. 2, 40 and 41, respectively, the following upper bound is obtained

$$
\begin{aligned}
& \dot{V}_{f} \leq--k_{f} m_{1}\|\eta\|^{2}-\|y\|^{2}-\|\operatorname{Tanh}(e)\|^{2} \\
&+\|\eta\|\left(\left(\xi_{1}+\xi_{7}\right)\|x\|+\xi_{2}\|y\|^{2}+\xi_{3}\|y\|^{3}\right. \\
&\left.+\xi_{4}\|y\|^{4}+\xi_{5}\|y\|^{5}+\xi_{6}\|\eta\|\|y\|\right)
\end{aligned}
$$

and substituting the control gain $k_{f}$ in Eq. 49, righthand side becomes

$$
\begin{aligned}
\dot{V}_{f} \leq & -\|\eta\|^{2}-\|y\|^{2}-\|\operatorname{Tanh}(e)\|^{2} \\
& +\left(\left(\xi_{1}+\xi_{7}\right)\|\eta\|\|x\|-k_{n}\left(\xi_{1}+\xi_{7}\right)^{2}\|\eta\|^{2}\right) \\
& +\left(\xi_{2}\|\eta\|\|y\|^{2}-2 n \xi_{2}^{2}\|\eta\|^{2}\right) \\
& +\left(\xi_{3}\|\eta\|\|y\|^{3}-2 n^{2} \xi_{3}^{2}\|\eta\|^{2}\right) \\
& +\left(\xi_{4}\|\eta\|\|y\|^{4}-2 n^{3} \xi_{4}^{2}\|\eta\|^{2}\right) \\
& +\left(\xi_{5}\|\eta\|\|y\|^{5}-2 n^{4} \xi_{5}^{2}\|\eta\|^{2}\right) \\
& +\left(\xi_{6}\|\eta\|^{2}\|y\|-\xi_{6} \sqrt{n}\|\eta\|^{2}\right) .
\end{aligned}
$$

After completing the squares for the bracketed terms, the following bound can be obtained

$$
\begin{aligned}
\dot{V}_{f} \leq & -\frac{1}{2}\|x\|^{2}-\frac{1}{2}\|\eta\|^{2}-\frac{1}{2}\|\operatorname{Tanh}(e)\|^{2}+\frac{1}{4 k_{n}}\|x\|^{2} \\
& +\frac{1}{2}\|y\|^{2}\left(\frac{1}{4 n}\|y\|^{2}+\frac{1}{4 n^{2}}\|y\|^{4}+\frac{1}{4 n^{3}}\|y\|^{6}\right. \\
& \left.+\frac{1}{4 n^{4}}\|y\|^{8}-1\right) \\
& -\xi_{6} \sqrt{n}\|\eta\|^{2}\left(1-\frac{\|y\|}{\sqrt{n}}\right) .
\end{aligned}
$$

Since $\|y\|^{2 l} \leq n^{l} \forall t, \dot{V}$ can be bounded in the following sense

$\dot{V}_{f} \leq-\beta\|x\|^{2}$ 
where $\beta$ is a positive constant defined as

$\beta \triangleq \frac{1}{2}-\frac{1}{4 k_{n}}$.

Since $\|y\|^{2} \leq n \max \left(y_{i}^{2}\right)$, the region $S_{1}$ defined in Eq. 48 is obtained, so,

$\dot{V}_{f} \leq-\beta\|x\|^{2}$ if $\left\{\eta, y, e, \tilde{\theta}, v_{\tilde{E}}, v_{\tilde{D}_{k}}\right\} \in S_{1}$.

As $S_{1} \subset S$, where $S$ was defined in Eq. 51, the region of attraction will contain the largest level set of $V_{f}(t)$ inside the set $S_{1}$. Since all level sets of $V_{f}(t)$ are contained inside $S$, then $S_{1}$ is invariant and is an estimate of the stability region. So, for initial conditions inside $S_{1}, V_{f}(t)$ is bounded, hence $\eta(t), \tilde{D}_{k}(t), \tilde{E}(t) \in \mathcal{L}_{\infty}^{n}$, and $\tilde{\theta}(t) \in \mathcal{L}_{\infty}^{p}$, therefore using signal chasing arguments we can claim that all signals in the closed loop error system are bounded and $x(t) \in \mathcal{L}_{\infty} \cap \mathcal{L}_{2}$ and $\dot{x}(t) \in \mathcal{L}_{\infty}$. Applying Barbalat's Lemma yields $\|x(t)\| \rightarrow 0$ as $t \rightarrow \infty$. Due to the structure of $x$ defined in Eq. 42, we can conclude that the signal $\|\eta\|,\|y\|$ and $\|$ Tanh $\|$ also converge to zero as time approaches to infinity. And finally using the properties of hyperbolic functions we can conclude that $\|e(t)\| \rightarrow 0$. Since no restrictions were imposed on initial conditions of position and velocity of the mechanical system, the result is global.

Remark 3 We want to point out that the condition given in Eq. 49 is only a sufficient condition that ensures that the convergence of the tracking error and the stability of the closed loop system. Selection of the corresponding gains when the upper bounds of the system uncertainties are not precisely known should be done with extreme caution. Small gain selection might not satisfy the given sufficient condition and extremely high gain selections might end up with performance degradation and even instability.

\subsection{Observer Based Approach}

In this section, an observer based approach will be utilized for the same problem. The development continues after (19).

The following velocity observer, denoted by $\dot{\hat{q}}(t) \in$ $\mathbb{R}^{n}$, is utilized in the controller design [4]

$$
\begin{aligned}
\dot{\hat{q}} & =p_{o}+K_{0} \tilde{q}-K_{c} e \\
\dot{p}_{o} & =K_{1} \operatorname{Sgn}(\tilde{q})+K_{2} \tilde{q}-\alpha K_{c} e
\end{aligned}
$$

where $p_{o}(t) \in \mathbb{R}^{n}$ is an auxiliary variable, $K_{0}, K_{c}$, $K_{1}, K_{2} \in \mathbb{R}^{n \times n}$ are positive definite, diagonal gain matrices, $\alpha \in \mathbb{R}$ is a positive gain and $\operatorname{Sgn}(\tilde{q}) \in \mathbb{R}^{n}$ is defined as

$\operatorname{Sgn}(\tilde{q}) \triangleq\left[\operatorname{sgn}\left(\tilde{q}_{1}\right) \operatorname{sgn}\left(\tilde{q}_{2}\right) \ldots \operatorname{sgn}\left(\tilde{q}_{n}\right)\right]^{T}$

where $\tilde{q}(t) \in \mathbb{R}^{n}$ is the position observation error defined as

$\tilde{q} \triangleq q-\hat{q}$.

To ease the presentation of the subsequent analysis, filtered tracking error $r(t) \in \mathbb{R}^{n}$ and filtered observation error $s(t) \in \mathbb{R}^{n}$ are defined as

$r \triangleq \dot{e}+\alpha e$ and $s \triangleq \dot{\tilde{q}}+\alpha \tilde{q}$.

Using Eqs. 62-66, the following observer dynamics is obtained

$\ddot{\hat{q}}=K_{1} \operatorname{Sgn}(\tilde{q})+K_{2} \tilde{q}+K_{0} \dot{\tilde{q}}-K_{c} r$.

Based on the subsequent error system development and stability analysis, the control input is designed as

$\tau=Y_{d} \hat{\theta}+K_{p} e+\alpha K_{c}\left(q_{d}-\hat{q}\right)+K_{c}\left(\dot{q}_{d}-\dot{\hat{q}}\right)+\hat{\gamma}$

where $\hat{\gamma}$ was previously defined in Eq. 21 and $K_{p} \in$ $\mathbb{R}^{n \times n}$ is a diagonal, positive definite gain matrix. Note that, the control input design in Eq. 68 can be rearranged as

$$
\begin{aligned}
\tau= & Y_{d} \hat{\theta}+K_{p} e+K_{c} r+K_{c} s+\hat{E} \operatorname{Tanh}(e) \\
& +\sum_{k=1}^{h} \hat{D}_{k} \operatorname{Sin}(k e)
\end{aligned}
$$

where Eqs. 13, 65 and 66 were utilized. The adaptive update rules for $\hat{\theta}(t), \hat{E}(t), \hat{D}_{k}(t)$ are generated according to

$$
\begin{aligned}
\hat{\theta}(t)= & \Gamma \int_{0}^{t}\left[\alpha Y_{d}^{T}(\sigma) e-\frac{d Y_{d}^{T}(\sigma)}{d \sigma} e(\sigma)\right] d \sigma \\
& +\Gamma Y_{d}^{T}(t) e(t)-\Gamma Y_{d}^{T}(0) e(0) \\
\hat{E}(t)= & \Psi \int_{0}^{t} v_{o}(\sigma) d_{\tanh }(\sigma) d \sigma+\Psi d_{\ln }(t)-\Psi d_{\ln }(0)
\end{aligned}
$$

$$
\begin{aligned}
\hat{D}_{k}(t)= & \Psi_{k} \int_{0}^{t} v_{o}(\sigma) d_{\sin }(\sigma) d \sigma-\frac{1}{k} \Psi_{k} d_{\cos }(t) \\
& +\frac{1}{k} \Psi_{k} d_{\cos }(0) \text { for } k=1,2, \ldots, h
\end{aligned}
$$


where $v_{o}(t) \in \mathbb{R}^{n \times n}$ is an auxiliary time-varying matrix defined as

$v_{o}(t) \triangleq \alpha \operatorname{diag}\left\{e_{1}(t), e_{2}(t), \ldots, e_{n}(t)\right\}$

and $d_{\tanh }(t), d_{\cos }(t), d_{\sin }(t)$ and $d_{\ln }(t)$ were previously defined in Eqs. 29-32, respectively, $\Gamma \in \mathbb{R}^{p \times p}, \Psi \in$ $\mathbb{R}^{n \times n}, \Psi_{k} \in \mathbb{R}^{n \times n}$ are constant, diagonal, positive definite adaptation gain matrices. Subsequent stability analysis requires $\hat{\theta}(t)$ and its time derivative to be bounded functions and thus the update rule in Eq. 70 is assumed to be generated by the use of a projection algorithm.

Using the observer dynamics in Eq. 67 and system dynamics in Eq. 1, the observation error dynamics is obtained as

$\ddot{\tilde{q}}=N-K_{1} \operatorname{Sgn}(\tilde{q})-K_{2} \tilde{q}-K_{0} \dot{\tilde{q}}+K_{c} r$

where Eq. 66 was utilized and $N(t) \in \mathbb{R}^{n}$ is an auxiliary term defined as

$$
N \triangleq M^{-1}(q)\left(\tau-V_{m}(q, \dot{q}) \dot{q}-G(q)-F_{d} \dot{q}-\tau_{d}\right) .
$$

After using the control input in Eq. 69, adding and subtracting the desired dynamics given in Eq. 7, the above auxiliary term can be segregated as some of two auxiliary terms

$N=N_{d}+N_{b}$

where the auxiliary terms $N_{d}(t), N_{b}(t) \in \mathbb{R}^{n}$ are defined as

$$
\begin{aligned}
N_{d} \triangleq & \ddot{q}_{d}-M^{-1}\left(q_{d}\right) Y_{d} \tilde{\theta} \\
N_{b} \triangleq & {\left[M^{-1}(q)-M^{-1}\left(q_{d}\right)\right]\left[M\left(q_{d}\right) \ddot{q}_{d}-Y_{d} \tilde{\theta}\right] } \\
& +M^{-1}(q)\left[V_{m}\left(q_{d}, \dot{q}_{d}\right) \dot{q}_{d}-V_{m}(q, \dot{q}) \dot{q}\right] \\
& +M^{-1}(q)\left[G\left(q_{d}\right)-G(q)+F_{d}\left(\dot{q}_{d}-\dot{q}\right)\right] \\
& +M^{-1}(q)\left[K_{p} e+K_{c} r+K_{c} s\right] \\
& -M^{-1}(q)\left[\tilde{E} \operatorname{Tanh}(e)+\sum_{k=1}^{h} \tilde{D}_{k} \operatorname{Sin}(k e)\right] .
\end{aligned}
$$

Using the boundedness properties of the desired trajectory and Property 1 , it can be shown that both $N_{d}$ and its time derivative are bounded. Furthermore, following a similar way in [4], it can be proven that $N_{b}$ can be upper bounded as

$$
\left\|N_{b}\right\| \leq \rho_{01}\|e\|+\rho_{02}\|r\|+\rho_{03}\|r\|^{2}+\rho_{04}\|s\|
$$

where $\rho_{01}, \rho_{02}, \rho_{03}, \rho_{04}$ are known positive bounding constants. In view of the segregation in Eq. 76, the filtered observer error dynamics can be obtained as

$\dot{s}=N_{d}+N_{b}-K_{1} \operatorname{Sgn}(\tilde{q})-K_{2} \tilde{q}-\left(K_{0}-\alpha I_{n}\right) \dot{\tilde{q}}+K_{c} r$.

If the observer gains are selected to satisfy

$\alpha\left(K_{0}-\alpha I_{n}\right)=K_{2}$

then, filtered observer error dynamics can be rearranged to have the following form

$\dot{s}=N_{d}+N_{b}-K_{1} \operatorname{Sgn}(\tilde{q})-\frac{1}{\alpha} K_{2} s+K_{c} r$.

The filtered error dynamics is obtained by using the system model in Eq. 1 and the error definitions in Eqs. 13 and 66 as

$M(q) \dot{r}=-V_{m}(q, \dot{q}) r+Y_{s} \theta+\gamma-\tau$

where $Y_{S}(t) \theta$ and $\gamma(t)$ terms are obtained from

$$
\begin{aligned}
Y_{s} \theta+\gamma= & M(q)\left(\ddot{q}_{d}+\alpha \dot{e}\right)+V_{m}(q, \dot{q})\left(\dot{q}_{d}+\alpha e\right) \\
& +G(q)+F_{d} \dot{q}+\tau_{d} .
\end{aligned}
$$

Utilizing the control input in Eq. 69, we obtain

$$
\begin{aligned}
M(q) \dot{r}= & -V_{m}(q, \dot{q}) r-K_{p} e-K_{c} r-K_{c} s+\varphi \\
& +\tilde{E} \operatorname{Tanh}(e)+\sum_{k=1}^{h} \tilde{D}_{k} \operatorname{Sin}(k e)+Y_{d} \tilde{\theta}
\end{aligned}
$$

where $\varphi(t) \in \mathbb{R}^{n}$ is defined as

$\varphi \triangleq Y_{s} \theta-Y_{d} \theta$.

The above auxiliary term can be bounded as [4]

$\|\varphi\| \leq \rho_{1}(\|e\|)\|e\|+\rho_{2}(\|e\|)\|r\|$

where $\rho_{1}(e)$ and $\rho_{2}(e)$ are known positive bounding functions.

Theorem 2 The controller in Eq. 68, observer in Eqs. 62 and 63 with the adaptive update rules in Eqs. 70-72 ensures semi-global asymptotic stability of the equilibrium in the sense that $\|e(t)\|,\|\dot{\tilde{q}}(t)\| \rightarrow$ 0 as $t \rightarrow \infty$ provided that the observer gains are selected to satisfy (81), and controller gain $K_{c}$ and observer gain $K_{2}$ are chosen to satisfy the following constraints

$$
\begin{aligned}
& K_{c}=1+\rho_{2}+k_{n} \rho_{1}^{2} \\
& K_{2}=\alpha\left(1+\rho_{04}+k_{n}\left(\rho_{01}^{2}+\rho_{02}^{2}+\rho_{03}^{2}\right)\right)
\end{aligned}
$$


where $k_{n} \in \mathbb{R}$ is a nonlinear damping gain selected to satisfy

$k_{n}>\frac{1}{2}\left(1+\frac{\lambda_{2}}{2 \lambda_{1}}\|z(0)\|^{2}\right)$

and the entries of the gain matrix $K_{1}$ satisfy

$K_{1 i}>\left\|N_{d i}(t)\right\|_{\infty}+\frac{1}{\alpha}\left\|\dot{N}_{d i}(t)\right\|_{\infty}$

and $z(t) \in \mathbb{R}^{((4+h) n+p+1)}$ is defined as

$z \triangleq\left[\begin{array}{lllllll}s^{T} & r^{T} & e^{T} & \sqrt{P_{0}} & \tilde{\theta}^{T} & v_{\tilde{E}}^{T} & v_{\tilde{D}_{k}}^{T}\end{array}\right]^{T}$

where $v_{\tilde{E}}(t)$ and $v_{\tilde{D}_{k}}(t)$ were previously defined in Eqs. 46 and 47 , respectively, and $\lambda_{1}, \lambda_{2} \in \mathbb{R}$ are defined as

$$
\begin{aligned}
\lambda_{1} \triangleq & \frac{1}{2} \min \left\{1, m_{1}, \lambda_{\min }\left(K_{p}\right), \lambda_{\min }\left(\Gamma^{-1}\right), \lambda_{\min }\left(\Psi^{-1}\right),\right. \\
& \left.\times \min _{k}\left[\lambda_{\min }\left(\Psi_{k}^{-1}\right)\right]\right\}
\end{aligned}
$$

$$
\begin{gathered}
\lambda_{2} \triangleq \frac{1}{2} \max \left\{2, m_{2}, \lambda_{\max }\left(K_{p}\right), \lambda_{\max }\left(\Gamma^{-1}\right), \lambda_{\max }\left(\Psi^{-1}\right),\right. \\
\left.\times \max _{k}\left[\lambda_{\max }\left(\Psi_{k}^{-1}\right)\right]\right\} .
\end{gathered}
$$

Proof To prove the above theorem, the following nonnegative scalar function, denoted by $V_{o}(t) \in \mathbb{R}$, is defined

$$
\begin{aligned}
V_{o}(t) \triangleq & \frac{1}{2} s^{T} s+P_{0}+\frac{1}{2} r^{T} M(q) r+\frac{1}{2} e^{T} K_{p} e \\
& +\frac{1}{2} \tilde{\theta}^{T} \Gamma^{-1} \tilde{\theta}+\frac{1}{2} \operatorname{tr}\left\{\tilde{E} \Psi^{-1} \tilde{E}\right\} \\
& +\frac{1}{2} \operatorname{tr}\left\{\sum_{k=1}^{h} \tilde{D}_{k} \Psi_{k}^{-1} \tilde{D}_{k}\right\}
\end{aligned}
$$

where the scalar auxiliary function $P_{o} \in \mathbb{R}$ is defined as

$$
P_{o} \triangleq \zeta_{o}-\int_{t_{o}}^{t} \omega_{o}(\sigma) d \sigma
$$

with $\omega_{o}(t) \in \mathbb{R}$ and non-negative constant $\zeta_{o}$ being defined as

$$
\begin{aligned}
& \omega_{o} \triangleq s^{T}\left(N_{d}-K_{1} \operatorname{Sgn}(\tilde{q})\right) \\
& \zeta_{o} \triangleq \sum_{i=1}^{n} K_{1 i}\left|\tilde{q}_{i}(0)\right|-\tilde{q}^{T}(0) N_{d}(0) .
\end{aligned}
$$

Following a similar analysis to that of $[3,4,20]$, it can be proven that $P_{o}(t)$ in Eq. 96 is always non-negative provided that the sufficient condition in Eq. 91 is satisfied. In view of this, $V_{o}(t)$ is a Lyapunov function. Following bounds can be obtained

$\lambda_{1}\|x\|^{2} \leq \lambda_{1}\|z\|^{2} \leq V_{o} \leq \lambda_{2}\|z\|^{2}$

where $x(t) \in \mathbb{R}^{3 n}$ is defined as

$x \triangleq\left[\begin{array}{lll}s^{T} & r^{T} & e^{T}\end{array}\right]^{T}$.

After taking the time derivative of Eq. 95 and using Eqs. 14, 66, 70, 82, time derivative of Eqs. 96, and 85, we obtain

$\dot{V}_{o}=s^{T}\left(N_{b}-\frac{1}{\alpha} K_{2} s\right)+r^{T}\left(\varphi-K_{c} r\right)-\alpha e^{T} K_{p} e$.

Utilizing the bounds in Eqs. 79 and 87 yields the following upper bound for the right-hand side of the above expression

$$
\begin{aligned}
\dot{V}_{o} \leq & -\|s\|^{2}-\|r\|^{2}-\|e\|^{2} \\
& +\left(\rho_{01}\|s\|\|e\|-k_{n} \rho_{01}^{2}\|s\|^{2}\right) \\
& +\left(\rho_{02}\|s\|\|r\|-k_{n} \rho_{02}^{2}\|s\|^{2}\right) \\
& +\left(\rho_{03}\|s\|\|r\|^{2}-k_{n} \rho_{03}^{2}\|s\|^{2}\right) \\
& +\left(\rho_{1}\|r\|\|e\|-k_{n} \rho_{1}^{2}\|r\|^{2}\right)
\end{aligned}
$$

provided that $\alpha$ and $K_{p}$ are chosen to satisfy $\alpha \lambda_{\min }\left\{K_{p}\right\}>1$. After completing the squares for the terms in the brackets, we obtain

$$
\begin{aligned}
\dot{V}_{o} \leq & -\left(1-\frac{1}{2 k_{n}}\right)\|e\|^{2}-\left(1-\frac{1}{4 k_{n}}-\frac{\|r\|^{2}}{4 k_{n}}\right)\|r\|^{2} \\
& -\|s\|^{2}
\end{aligned}
$$

which in view of Eq. 100 can be rewritten as

$\dot{V}_{o} \leq-\left[1-\frac{1}{2 k_{n}}\left(1+\frac{1}{2}\|x\|^{2}\right)\right]\|x\|^{2}$.

Provided the following condition holds

$1-\frac{1}{2 k_{n}}\left(1+\frac{1}{2}\|x\|^{2}\right)>0$

$\dot{V}_{o}$ is negative definite. After utilizing the lower bound in Eq. 99, the above expression can be restated as

$1-\frac{1}{2 k_{n}}\left(1+\frac{V_{o}(t)}{2 \lambda_{1}}\right)>0$. 
In view of the above expression, the upper bound for $\dot{V}_{o}$ is obtained as

$\dot{V}_{o} \leq-\beta\|x\|^{2}$ provided that $2 k_{n}>1+\frac{V_{o}(t)}{2 \lambda_{1}}$

where $\beta \in \mathbb{R}$ is some positive constant satisfying $0<\beta \leq 1$. Since $\dot{V}_{o}$ is negative semi-definite, after utilizing Eqs. 92 and 99, we can obtain

$\dot{V}_{o} \leq-\beta\|x\|^{2}$

provided that (90) is satisfied. Based on Eqs. 108, 99 and 100, we can conclude that $V_{o}(t) \in \mathcal{L}_{\infty}$. Therefore the $P_{0} \in \mathcal{L}_{\infty}, s(t), r(t), e(t), \tilde{D}_{k}(t), \tilde{E}(t) \in \mathcal{L}_{\infty}^{n}$, and $\tilde{\theta}(t) \in \mathcal{L}_{\infty}^{p}$. From the boundedness of the actual system parameters we can conclude that $\hat{D}_{k}(t), \hat{E}(t) \in$ $\mathcal{L}_{\infty}^{n}$ and $\hat{\theta} \in \mathcal{L}_{\infty}^{p}$ Standard signal chasing arguments can then be utilized to show boundedness of all the signals under the closed-loop operation. Specifically, it can straightforwardly be shown that $x(t), \dot{x}(t) \in$ $\mathcal{L}_{\infty}$. After integrating (107) from initial time to infinity, it is easy to see that $x(t) \in \mathcal{L}_{2}$. Barbalat's Lemma can then be utilized to prove that the position tracking error and the velocity observer error converge to the origin asymptotically.

Remark 4 We would like to note that, to our best knowledge, all of the observer based output feedback controllers in the literature achieve semi-global stability result. However, aside from a theoretical weakness, by adjusting the controller gains accordingly the size of the region of attraction can be made arbitrary large.

Remark 5 For both of the output feedback adaptive controllers proposed here asymptotic tracking error convergence and the boundedness of the parameter estimations are guaranteed. However with the frame (a)

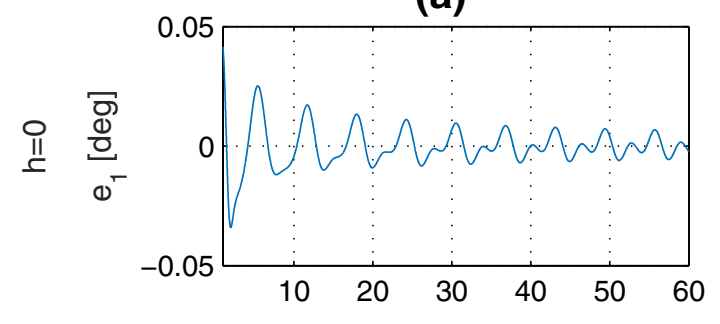

(c)

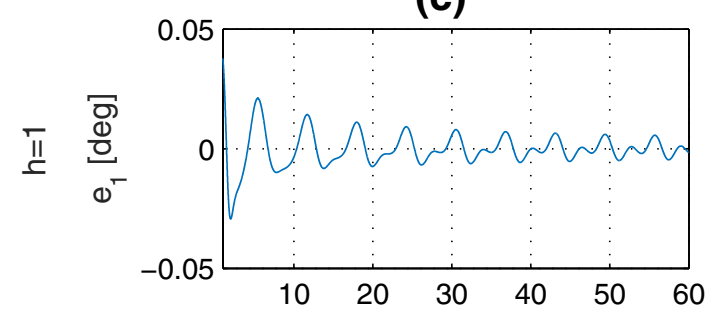

(e)

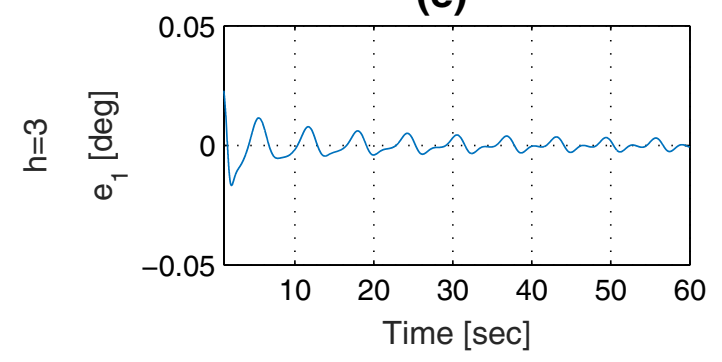

(b)

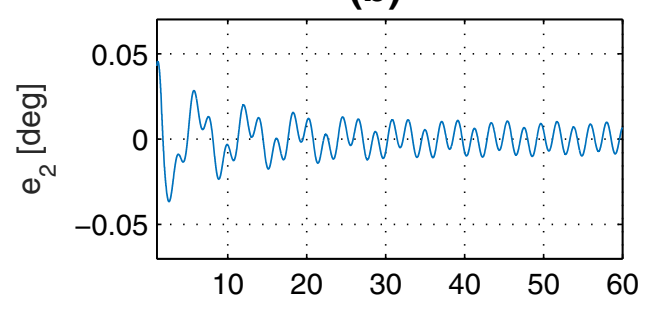

(d)

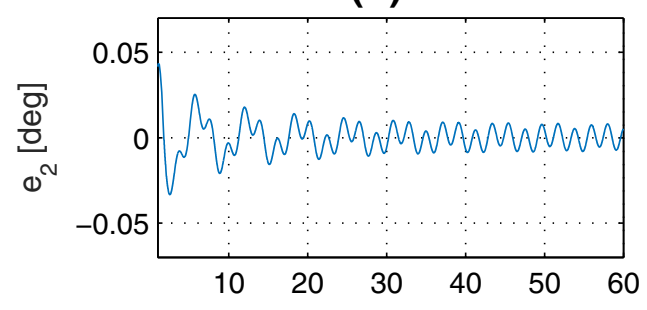

(f)

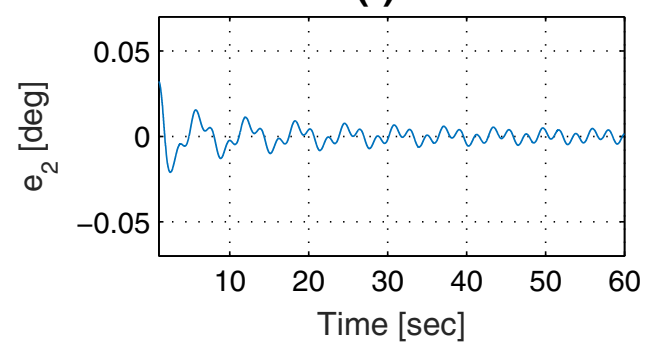

Fig. 1 Tracking error performances for the filter based OFB controller with $h=0, h=1$ and $h=3$ 
Fig. 2 Dynamical parameter estimates

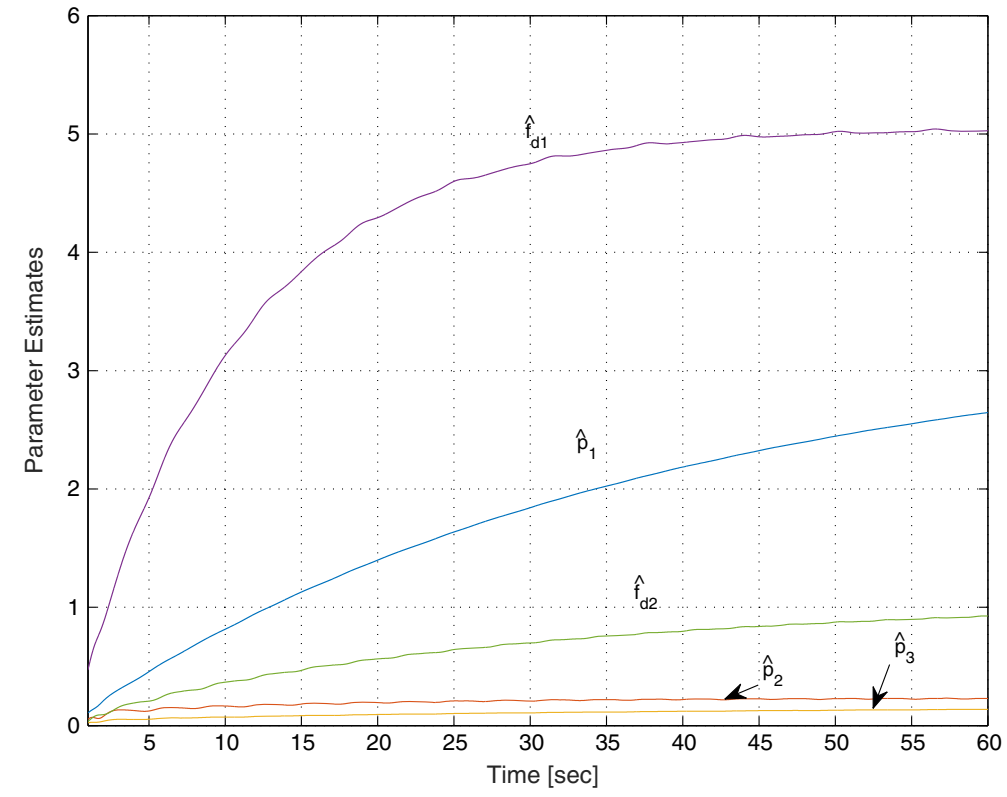

(a)

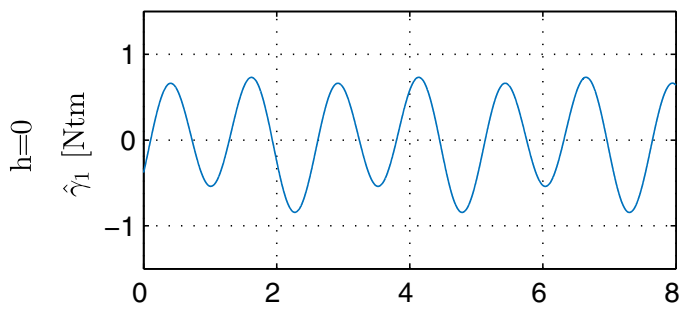

(c)

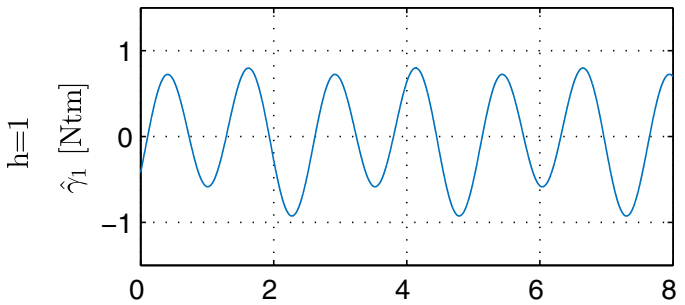

(e)

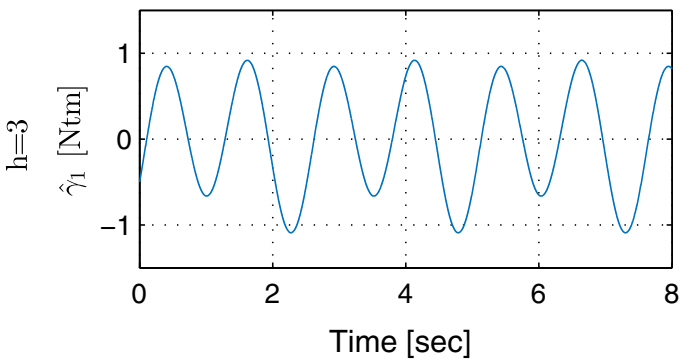

(b)

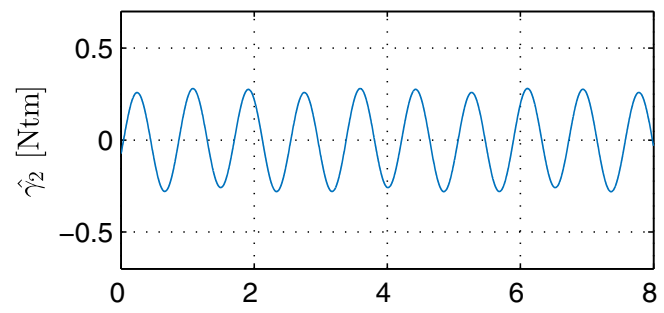

(d)

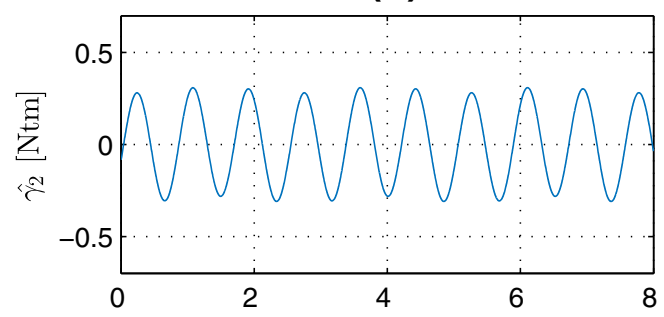

(f)

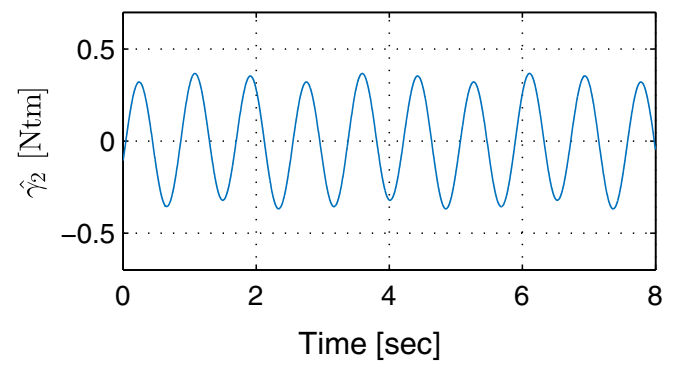

Fig. 3 The overall disturbance estimates at steady state for h values 0,1 and 3 (Filter based controller) 
(a)

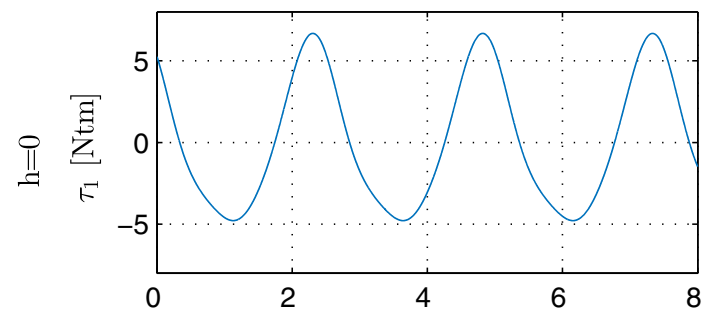

(c)

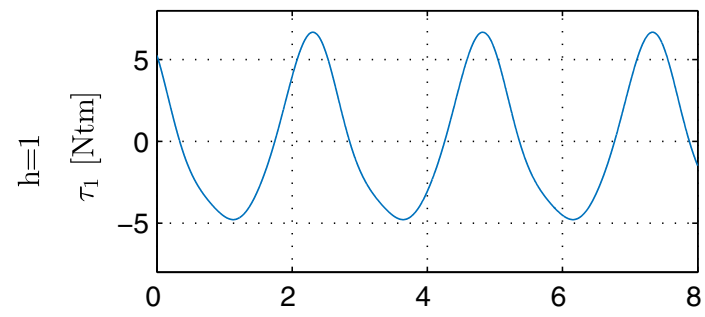

(e)

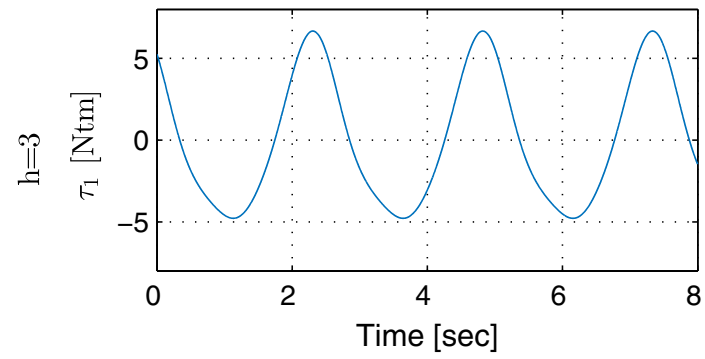

(b)

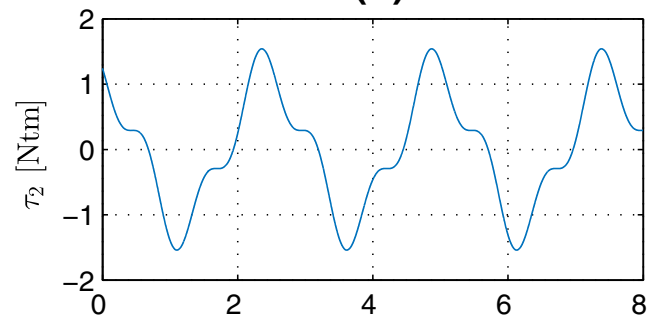

(d)

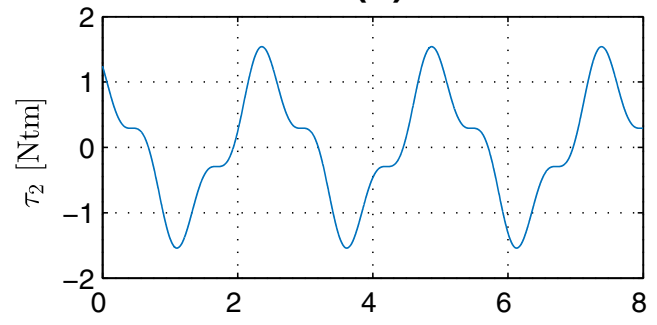

(f)

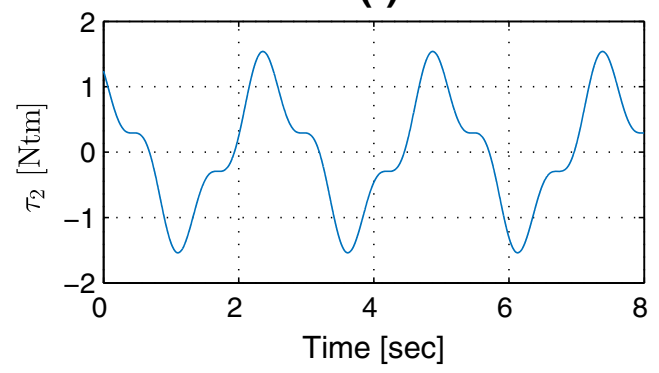

Fig. 4 The controller torque inputs $h$ values 0,1 and 3 (Filter based controller)

work proposed we were not able to ensure the convergence of the parameter estimates to their actual values or define a finite time for convergence of the parameter estimates. For the convergence of the parameters to their actual values some sort of (either for the actual states or for the desired state) persistency of excitation condition is required. For finite time parameter estimation convergence readers are referred to [21] where a full-state feedback robust controller with an adaptive parameter estimation rule is proposed.

\section{Numerical Studies}

The performances of the proposed adaptive output feedback controllers were simulated on a two link,

Table 1 Comparison table for the filtered based OFB controller

\begin{tabular}{lllll}
\hline & & $\max \left\{\left|e_{i}(t)\right|\right\}$ & $\max \left\{\left|\tau_{i}(t)\right|\right\}$ & $\mathcal{L}_{2}$ norm of $e_{i}(t)$ \\
\hline $\mathrm{h}=0$ & Link 1 & 0.8548 & 7.9726 & 1383.2 \\
& Link 2 & 1.5431 & 1.6131 & 9267 \\
$\mathrm{~h}=1$ & Link 1 & 0.7552 & 7.9204 & 948.12 \\
& Link 2 & 1.3232 & 1.5714 & 6963.9 \\
$\mathrm{~h}=3$ & Link 1 & 0.463 & 8.038 & 284.01 \\
& Link 2 & 0.7565 & 1.8062 & 2545.2 \\
\hline
\end{tabular}


direct-drive planar robot manipulator having the following dynamic model [22]

$$
\begin{aligned}
M(q) & =\left[\begin{array}{cc}
p_{1}+2 p_{3} \cos \left(q_{2}\right) & p_{2}+p_{3} \cos \left(q_{2}\right) \\
p_{2}+p_{3} \cos \left(q_{2}\right) & p_{2}
\end{array}\right] \\
V_{m}(q, \dot{q}) & =\left[\begin{array}{cc}
-p_{3} \sin \left(q_{2}\right) \dot{q}_{2} & -p_{3} \sin \left(q_{2}\right)\left(\dot{q}_{1}+\dot{q}_{2}\right) \\
p_{3} \sin \left(q_{2}\right) \dot{q}_{1} & 0
\end{array}\right] \\
F_{d} & =\left[\begin{array}{cc}
f_{d 1} & 0 \\
0 & f_{d 2}
\end{array}\right]
\end{aligned}
$$

where $p_{1}=3.473\left[\mathrm{~kg}-\mathrm{m}^{2}\right], p_{2}=0.193\left[\mathrm{~kg}-\mathrm{m}^{2}\right]$, $p_{3}=0.242\left[\mathrm{~kg}-\mathrm{m}^{2}\right], f_{d 1}=5.3[\mathrm{Nm}-\mathrm{sec}], f_{d 2}=$ $1.1[\mathrm{Nm}-\mathrm{sec}]$. Based on Eqs. 7 and 109-111, the constant parameter vector can be constructed as

$\theta=\left[\begin{array}{lllll}p_{1} & p_{2} & p_{3} & f_{d 1} & f_{d 2}\end{array}\right]^{T}$.

Following desired position trajectory was utilized

$q_{d}(t)=\left[\begin{array}{c}0.7 \sin (t)\left(1-\exp \left(-0.3 t^{3}\right)\right) \\ 1.2 \sin (t)\left(1-\exp \left(-0.3 t^{3}\right)\right)\end{array}\right][\mathrm{rad}]$

(a)

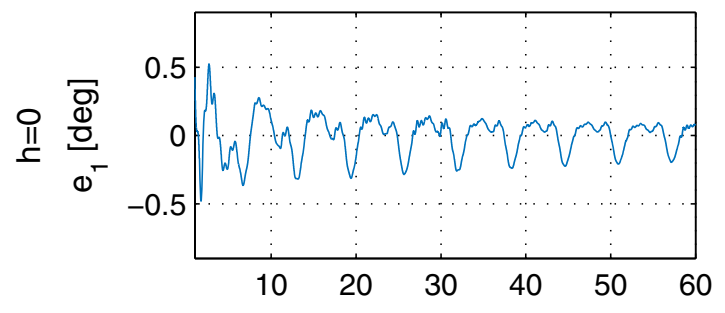

(c)

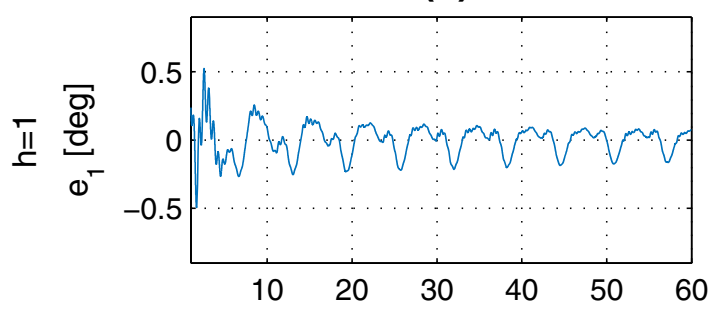

(e)

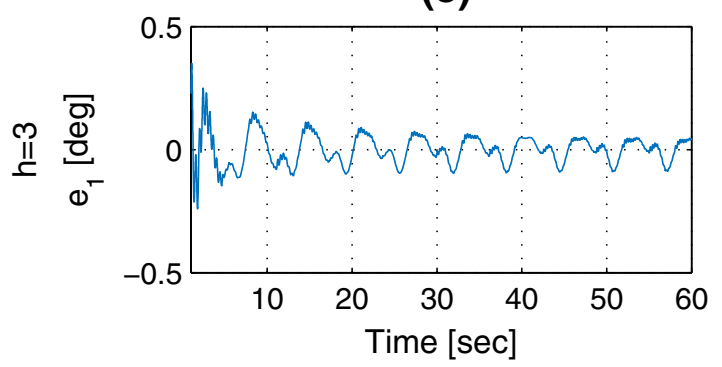

where the exponential term was included to ensure $e(0)=\dot{e}(0)=\ddot{e}(0)=0_{2 \times 1}$. In order to evaluate the performance of periodic disturbance estimation, the following periodic disturbance was added to the robot dynamics

$\tau_{d}(t)=\left[\begin{array}{l}\sin (2 t) \\ 0.4 \sin (3 t)\end{array}\right][N m]$.

For both sets of simulations (filter based and observer based), three levels of harmonic limit, $h$ defined in Eq. 15, are applied for disturbance estimation. For $h=0$ no estimation for the disturbance term is present in the controller, for $h=1$ only the first harmonic and for $h=3$ the first three harmonics are inserted in to the controller signal. The effects on the output are then presented in corresponding tables. The parameter estimates and system states were initialized to zero and the simulations were performed at a sampling frequency of $2 \mathrm{KHz}$.

(b)

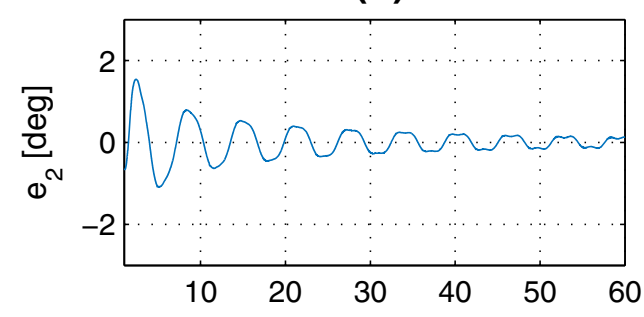

(d)

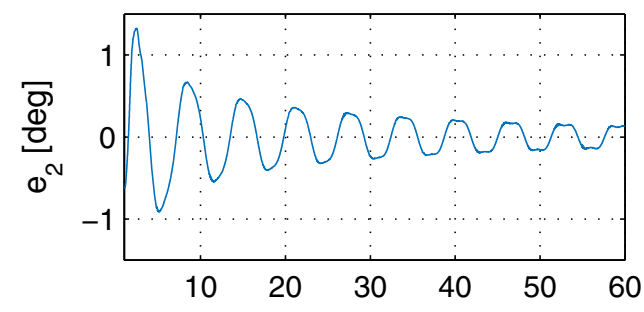

(f)

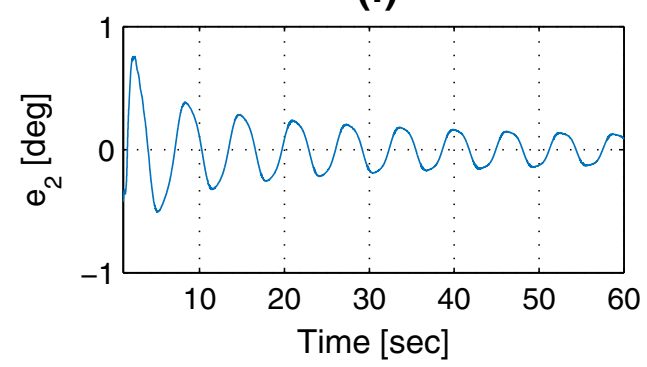

Fig. 5 Tracking error performance for the observer based OFB controller for $h$ values of 0, 1 and 3 


\subsection{Simulation Results for Filter Based Controller}

The first set of simulations were performed for the filter based controller proposed in Eq. 20, with the online adaptations defined in Eqs. 26, 27 and 28. The gain tuning process was done by trial and error method. The best performance was obtained when nonlinear filter based controller gains and adaptation update gains were selected as:

$$
\begin{aligned}
k_{f} & =\operatorname{diag}\{260,84.64\}, \\
\Psi & =500000 \times \operatorname{diag}\{10,1\}, \\
\Psi_{k} & =500000 \times \operatorname{diag}\{10,1\} \\
\Gamma & =\operatorname{diag}\{12.6,4.86,2.26,86.26,12.4\}
\end{aligned}
$$

Three sets of simulations were performed for $h=$ $0, h=1$, and $h=3$. The tracking error performance of the controllers are presented in Fig. 1. Dynamical

(a)

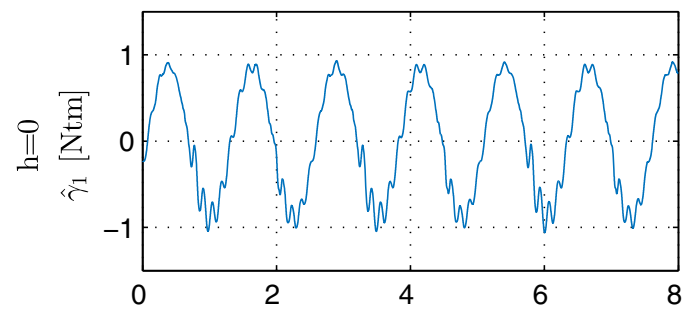

(c)

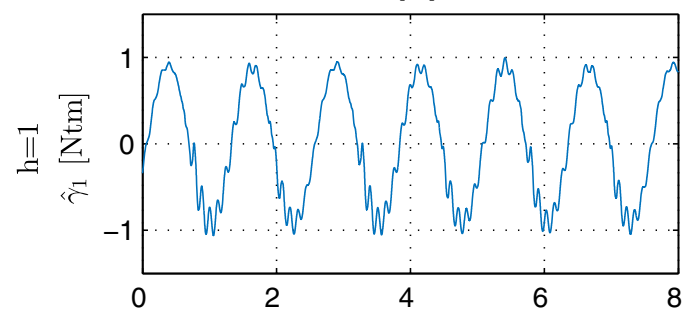

(e)

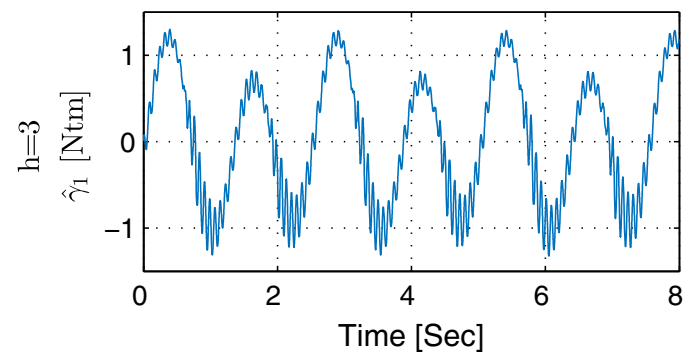

parameter estimates are presented in Fig. 2 (since parameter estimation plots were similar for all simulations, only one of them is presented here). Figures 3 and 4 show the total disturbance estimations, $\hat{\gamma}(t)$, and the controller torque input to the system.

Finally, Table 1 provides a comparison of maximum values of tracking error, input torque and $\mathcal{L}_{2}$ norm of the tracking error signals for $\mathrm{h}$ values 0,1 and 3.

From Table 1 and Figs. 1, 3 and 4, it is evident that the tracking error performance is increasing as the values of $h$ increases, and the control torque values remain in at comparable values. The increased tracking performance is more evident when the $\mathcal{L}_{2}$ norm of the tracking error signals are taken into account (last column of Table 1). The more the value of $h$ is increased the better tracking error performance becomes and the amount of control efforts are still at comparable values. (b)

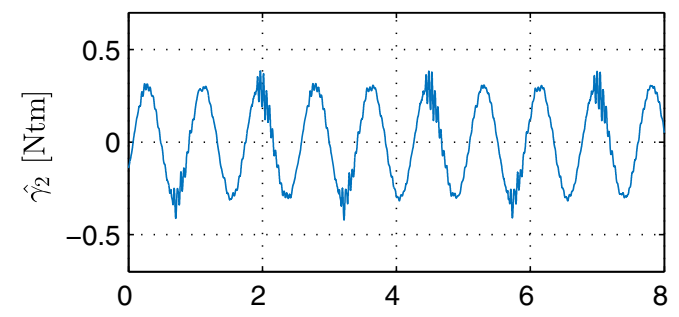

(d)

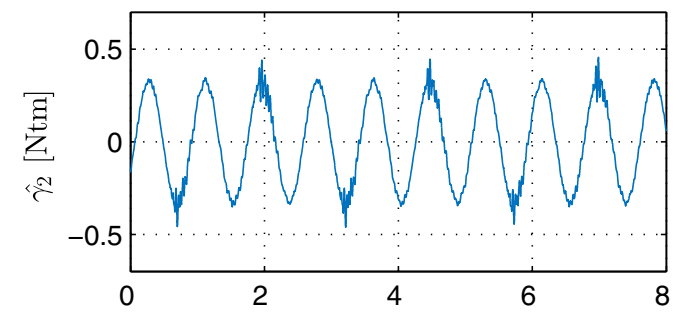

(f)

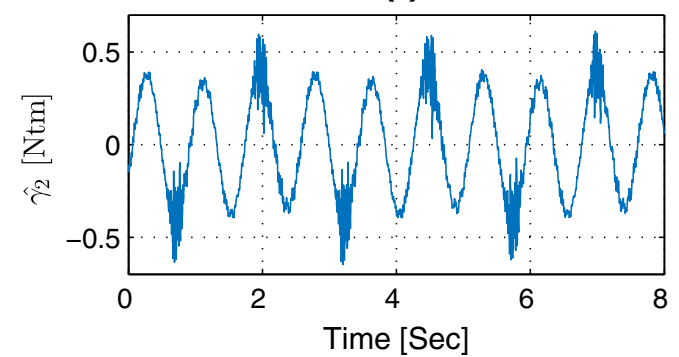

Fig. 6 The overall disturbance estimates at steady state for h values 0,1 and 3 (Observer based controller) 


\subsection{Simulation Results for Observer Based Controller}

The same simulations performed for the filtered based OFB (Output Feedback) controller are then performed for the observers based OFB controller presented in Eqs. 68, 62 and 63 with the adaptive update rules given in Eqs. 70-72. Similar to the set of simulations performed for the filtered based OFB controller 3 sets of simulations were performed. For all of the simulations in this set the contorller/observer gains and the adaptations are set to

$$
\alpha=3 \text {, }
$$$$
K_{0}=K_{1}=K_{2}=\operatorname{diag}\{10,8\}
$$

$K_{c}=\operatorname{diag}\{8,5\}, K_{p}=\operatorname{diag}\{80,75\}$

$\Psi=50000 \times \operatorname{diag}\{10,1\}, \Psi_{k}=50000 \times \operatorname{diag}\{10,1\}$

$\Gamma=\operatorname{diag}\{11.6,1.8,2.4,7.8,8.6\}$.

Note that the entries of the controller gain matrix , $K_{c}$, are selected as constants. In our case, this

(a)

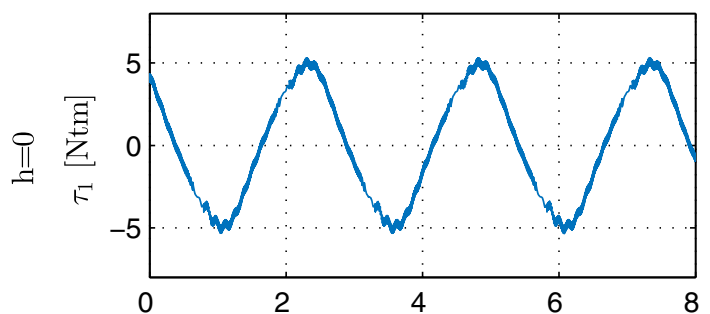

(c)

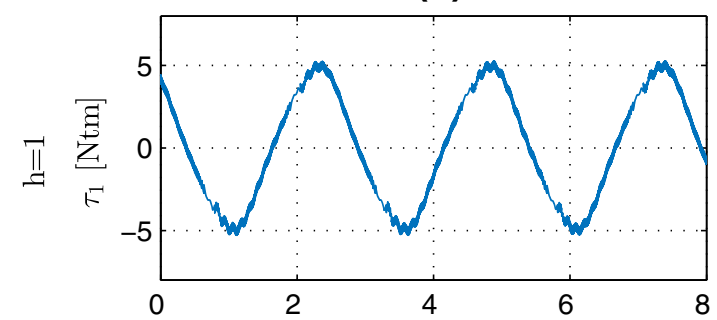

(e)

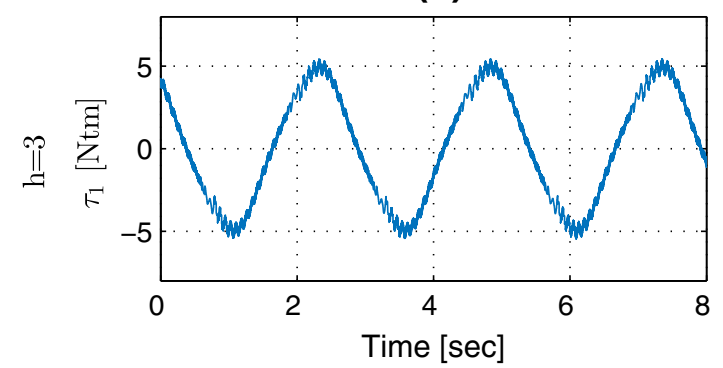

is mainly done to reduce the computational burden of calculating the bounding functions $\rho_{1}(\|e\|)$ and $\rho_{2}(\|e\|)$, defined in Eq. 87 on-line. However, we also want to note that on actual implementations instead of using bounding functions bounding constant values are preferred due to the practical reasons (ease of constant gain tuning as opposed to function tuning).

The tracking error performance of the controllers are presented in Fig. 5 while Figs. 6 and 7 present the total disturbance estimations, $\hat{\gamma}(t)$, and the controller torque input to the system respectively.

And finally Table 2 shows the comparison of maximum values of tracking error, input torque and $\mathcal{L}_{2}$ norm of the tracking error signals for $\mathrm{h}$ values 0,1 and 3.

Similar to the filtered based OFB controller, we can conclude from Table 2 and the corresponding simulation outputs that increasing the values of $h$ has a positive effect on the tracking error performance and (b)

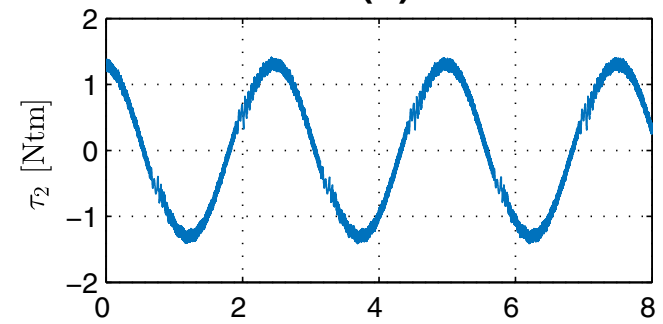

(d)

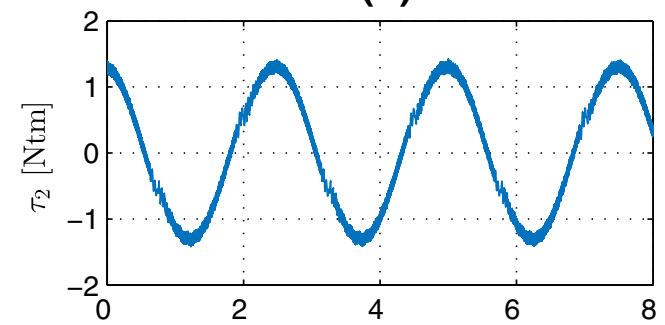

(f)

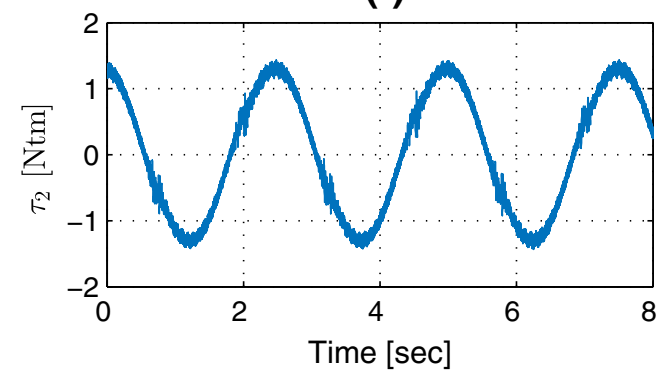

Fig. 7 The controller torque inputs at steady state h values 0,1 and 3 (Observer based controller) 
Table 2 Comparison table for the observer based OFB controller

\begin{tabular}{lllll}
\hline & & $\max \left\{\left|e_{i}(t)\right|\right\}$ & $\max \left\{\left|\tau_{i}(t)\right|\right\}$ & $\mathcal{L}_{2}$ norm of $e_{i}(t)$ \\
\hline $\mathrm{h}=0$ & Link 1 & 0.0430 & 6.6819 & 4.8579 \\
& Link 2 & 0.0454 & 1.6060 & 6.8927 \\
$\mathrm{~h}=1$ & Link 1 & 0.0398 & 6.6809 & 3.5970 \\
& Link 2 & 0.0433 & 1.6060 & 5.4749 \\
$\mathrm{~h}=3$ & Link 1 & 0.0266 & 6.6778 & 1.2538 \\
& Link 2 & 0.0323 & 1.6058 & 2.2551 \\
\hline
\end{tabular}

the control torque input to the system is not effected much.

\section{Conclusion}

In this paper, we have presented a velocity independent disturbance estimation methodology that can be used with both observer based and filtered based adaptive output feedback controllers. Specifically two adaptive output feedback controllers fused with online disturbance estimation for Euler-Lagrange systems. Following the two general classes of output feedback controllers in the literature, the first one is a nonlinear filter based adaptive output feedback controller, and the second is an observer based one. In order to improve the controller performances, the disturbance term in the Euler-Lagrange formulation together with the unstructured dynamic uncertainties (that is usually not linearly parameterizable) was modeled using a time-dependent Fourier series expansion with uncertain mean values and uncertain parameters for contribution of different frequencies. This modeling approach was fused with both controllers where the estimates of the periodic uncertainty modeling parameters were designed through Lyapunovtype synthesis and analysis tools. It was proven that the first controller ensured globally asymptotically stable tracking results, while the second controller is proven to be semi-globally asymptotically stable. Numerical simulation results were presented to illustrate the tracking performances of both controllers.

Future work will concentrate on extending the results presented here to robust and learning type output feedback controllers.

\section{References}

1. de Queiroz, M.S., Dawson, D.M., Nagarkatti, S., Zhang, F.: Lyapunov-Based Control of Mechanical Systems, ISBN: 978-0-8176-4086-6. Birkhuser Basel (2000)

2. Arimoto, S., Parra-Vega, V., Naniwa, T.: A class of linear velocity observers for nonlinear mechanical systems. In: Proc. Asian Control Conf., pp. 633-636. Tokyo (1994)

3. Xian, B., de Queiroz, M.S., Dawson, D.M., McIntyre, M.L.: Output feedback variable structure-like control of nonlinear mechanical systems. In: Proc. of IEEE Int. Conf. on Decision and Control, pp. 368-373 .Maui, Hawaii, USA (2003)

4. Zergeroglu, E., Tatlicioglu, E.: Observer based adaptive output feedback tracking control of robot manipulators. In: IEEE Int. Conf. on Decision and Control, pp. 3638-3643. Atlanta (2010)

5. Zergeroglu, E., Dawson, D.M., de Queiroz, M.S., Krstic, M.: On global output feedback control of robot manipulators. In: IEEE Int. Conf. on Decision and Control, pp.50735078. Sydney (2000)

6. Burg, T.C., Dawson, D.M., Vedagarbha, P.: A redesigned DCAL controller without velocity measurements: Theory and demonstration. Robotica 15, 337-346 (1997)

7. Zergeroglu, E., Dixon, W.E., Haste, D., Dawson, D.M.: A composite adaptive output feedback tracking controller for robotic manipulators. Robotica 17, 591-600 (1999)

8. Delibasi, A., Zergeroglu, E., Kucukdemiral, E.B., Cansever, G.: Adaptive self-tuning control of robot manipulators with periodic disturbance estimation. Int. J. Robot. Autom. 25(1), 48-56 (2010)

9. Liaw, C.M., Lin, F.J.: Position control with fuzzy adaptation for induction servo drives. IEEE Proc. Electr. Power. Appl., 397-404 (1995)

10. Tsay, D.L., Chung, H.Y., Lee, C.J.: Adaptive control of nonlinear systems using Sugeno type of fuzzy logic. IEEE Tr. Fuzzy Syst. 17, 225-229 (1999)

11. Wai, R.J., Lin, C.M., Hsu, C.F.: Adaptive fuzzy sliding mode control for electical servo drive. Fuzzy Sets Syst. 143, 295-310 (2004)

12. Lim, S.Y., Dawson, D.M., Anderson, K.: Re-examining the Nicosia-Tomei robot observer-controller from a 
backstepping perspective. IEEE Tr. Control Syst. Technol. 4(3), 304-310 (1996)

13. Yuan, J., Stepanenko, Y.: Robust control of robotic manipulators without velocity measurements. Int. J. Robust Nonlinear Control 1, 203-213 (1991)

14. Berghuis, H., Nijmeijer, H.: Robust control of robotic manipulators without velocity measurements. IEEE Tr. Autom. Control 39(10), 740-754 (1993)

15. Lewis, F.L., Abdallah, C.T., Dawson, D.M.: Control of Robot Manipulators. Macmillan Publishing Co., New York (1993)

16. Lewis, F.L., Dawson, D.M., Abdallah, C.T.: Robot Manipulator Control: Theory and Practice. Marcel Dekker, Inc., New York (2004). ISBN: 0-8247-4072-6

17. Tomei, P.: Adaptive PD controller for robot manipulators. IEEE Tr. Robot. Autom. 7(4), 565-570 (1991)

18. Nicosia, S., Tomei, P.: Robot control by using only position measurements. IEEE Tr. Autom. Control 35(9), 1058-1061 (1990)

19. Sadegh, N., Horowitz, R.: Stability and robustness analysis of a class of adaptive controllers for robot manipulators. Int. J. Robot. Res. 9(3), 74-92 (1990)

20. Xian, B., Dawson, D.M., de Queiroz, M.S., Chen, J.: A continuous asymptotic tracking control strategy for uncertain nonlinear systems. IEEE Tr. Autom. Control 47(7), 12061211 (2004)

21. Na, J., Mahyuddin, M.N., Herrmann, G., Ren, X., Barber, P.: Robust adaptive finite-time parameter estimation and control of robotic systems. Int. J. Robust Nonlinear Control 25, 3045-307192 (2015)

22. Direct Drive Manipulator Research and Development Package Operations Manual. Integrated Motion Inc., Berkeley, (1992)

Orhan Aksoy received his B.S. and M. S. degrees in Electrical and Electronics Engineering from Hacettepe University (Turkey, 1993) and Middle East Technical University (Turkey, 1996), respectively. Afterwards, he has been employed in various engineering companies in USA and Turkey, mostly working on ground vehicle simulator software. Since 2005, he has been working in HAVELSAN A.S. on naval combat management systems. Since 2010, he has been studying at Gebze Technical University for his Ph.D. degree in Computer Science Department on nonlinear adaptive and output feedback systems and optimal approaches.
Erkan Zergeroglu received his B.Sc. and M.Sc. degrees in Electrical and Electronic Engineering from Hacettepe University and Middle East Technical University in 1992 and 1996, respectively, both in Turkey. In 2000 he received his Ph.D. degree in Electrical Engineering from Clemson University, South Carolina, USA. He then joined Lucent Technologies-Bell Labs Innovations, Optical Fiber Solutions, Sturbridge MA as Member of Technical Stuff from 2000-2002. In 2002, he joined his Computer Engineering Department of Gebze Institute of Technology (which in 2015 renamed as Gebze Technical University) Kocaeli-Turkey, where he currently leads the Control Technologies and Robotics Laboratory.

His main research interests are in the fields of nonlinear model-based robust, adaptive, and learning control with application to electro-mechanical systems, including but not limited to robotics and control of robot manipulators, visual servoing applications, and variable speed wind turbines.

Enver Tatlicioglu received the B.Sc. degree in Electrical \& Electronics Engineering from Dokuz Eylul University, Izmir, Turkey and the Ph.D. degree in Electrical and Computer Engineering from Clemson University, Clemson, SC, USA in 1999 and 2007, respectively. Upon completion of his Ph.D. degree, he worked as a post-doctoral research fellow in the Department of Electrical and Computer Engineering at Clemson University then he joined the Department of Electrical \& Electronics Engineering at Izmir Institute of Technology, Izmir, Turkey where he is currently an associate professor.

His research interests include control and identification of time delay systems, dynamic modelling of extensible continuum robot manipulators, non-linear control techniques for kinematically redundant robot manipulators, partial state feedback and output feedback control, haptic systems and teleoperation; learning, robust and adaptive control of non-linear systems. 